Meta

Journal des traducteurs

Translators' Journal

\title{
Choix d'équivalents anglais-français en recherche documentaire
}

\section{Robert Serré}

Volume 21, numéro 2, juin 1976

URI : https://id.erudit.org/iderudit/004109ar

DOI : https://doi.org/10.7202/004109ar

Aller au sommaire du numéro

Éditeur(s)

Les Presses de l'Université de Montréal

ISSN

0026-0452 (imprimé)

1492-1421 (numérique)

Découvrir la revue

Citer cet article

Serré, R. (1976). Choix d'équivalents anglais-français en recherche documentaire. Meta, 21(2), 147-155. https://doi.org/10.7202/004109ar d'utilisation que vous pouvez consulter en ligne.

https://apropos.erudit.org/fr/usagers/politique-dutilisation/ 


\section{CHOIX D'ÉQUIVALENTS ANGLAIS-FRANCAIS EN RECHERCHE DOCUMENTAIRE}

Les sources sont abrégées entre parenthèses après chaque équivalent; les références bibliographiques sont énumérées à la suite de la liste d'équivalents, en regard des abréviations correspondantes.

\section{ABSTRACT JOURNAL}

- journal de résumés (Unisist) ;

- revues signalétiques (VIBANQUE, Université Laval, 1973) ;

- bulletins de résumés, bulletins d'analyses, revues de résumés (Van Dijk, 1969) ;

- les autres revues bibliographiques étrangères telles que Chemical Abstracts (BS 101, Suppl. 1975).

\section{ALER'TING SERVICE (voir CURRENT AWARENESS)}

\section{BATCH PROCESSING (voir aussi TIME-SHARING)}

- traitement par lots, par trains, traitement séquentiel, différé, groupé (Ginguay, 1972) ;

- (travail en) temps réservé (CETTF, 1972) ;

- traitement par lots : mode de traitement de l'information suivant lequel les programmes à exécuter ou les données à traiter sont groupés en lots (BT 155);

- les recherches se font en temps différé (Dusoulier, 1971).

CITATION (parfois utilisé dans le sens de RÉFÉRENCE)

\section{CITATION INDEX}

- index de citations (Unisist) ;

- l'index des citations sert à repérer tous les articles où l'on cite un document donné (Bonnelly, 1973) ;

- l'index des citations [...] l'article citant un article original est identifié par une citation source, l'article cité par des citations références (Chaumier, 1971).

\section{CLEARING HOUSE (voir aussi DEPOSITORY)}

- dépôt, centre (de collecte et) de distribution, organisme de distribution, centre de dépôt (Unisist) ;

- bureau central d'information : agence centrale chargée de recueillir, de classifier et de diffuser l'information, ou de coordonner l'activité des centres d'information (VTBB, 1969) ;

- des centrales de regroupement et de diffusion de cette littérature insaisissable (Chaumier, 1971).

\section{COMPUTERIZED INFORMATION RETRIEVAL}

- la recherche documentaire automatisée (Marlot, 1973);

- centres ou services de documentation mécanisés (Chaumier, 1971) ;

- système, mode de recherche documentaire, de documentation automatisée (AT oct. 1974) ; 
- documentation automatique (PASCAL, CNRS (Paris), 1974);

- système de documentation automatisé (le Monde).

\section{CONTROLLED VOCABULARY (voir WORD AUTHORITY LIST)}

\section{CONVERSATIONAL (aussi INTERACTIVE)}

- conversationnel (Ginguay, 1972) ;

- terminal de dialogue, mode « dialogue » (Ginguay, 1972);

- en conversationnel (Dusoulier, 1971);

- de dialogue, dialogué : permettant un dialogue entre l'ordinateur et l'utilisateur (BT 155).

\section{CURRENT AWARENESS}

- services parutions récentes (le Monde, 1971) ;

- une information permanente (le Monde, 1972) ;

- liste d'information courante, journal d'information courante (Unisist) ;

- une documentation permanente (SODEXPORT, France, 1972) ;

- la signalisation courante de la documentation (Bonnelly, 1973);

- mise au courant de l'actualité scientifique (ISTC II-3, 1970) ;

- services mensuels de signalement personnalisés (Poincaré, 1973) ;

- système sélectif d'information courante (BS 101, 1970).

\section{DATA BANK}

- banque de données (Ginguay, 1972) ;

- banque de données : ensemble de collections de données, c'est-à-dire de fichiers voisins ou apparentés (BT 155).

\section{DATA BASE}

- base (commune) de données ; fichier central (Ginguay, 1972) ;

- base de données (BS 101, 1971);

- base de données : collection de données homogènes et tendant à la même fin (BT 155).

\section{DEPOSITORY (voir aussi CLEARING HOUSE)}

- dépôt, organisme central de collecte (Unisist) ;

- bibliothèque de conservation : bibliothèque dont la fonction principale consiste à conserver la documentation qu'elle acquiert, comme les bibliothèques nationales (VTBB, 1969) ;

— bibliothèque de dépôt : bibliothèque légalement désignée pour recevoir gratuitement les publications officielles (VTBB, 1969).

\section{DISPLAY}

- présentation sur écran cathodique (Unisist) ;

- la rapidité de l'affichage ; terminaux avec claviers et écrans de visualisation (Mathelot, 1969);

- affichage, visualisation, signalisation (Ginguay, 1972) ;

- visuel, console de visualisation : dispositif d'affichage ou d'inscription sur un écran ou une console à tube cathodique ; cet écran ou cette console (BT 155). 


\section{FEEDBACK}

- mécanismes de réponse (Unisist) ;

- un contrôle de retour qui fait connaître le niveau de satisfaction des usagers (Chaumier, 1971).

\section{FIELD}

- zone (de mémoire, de carte, d'instruction, etc.) (Ginguay, 1972) ;

- zone adresse (d'une instruction) (Ginguay, 1972) ;

- chaque zone du résumé (zone-titre, zone-auteur, zone-source, zone-résumé, etc.) (Documentaliste, 1971);

- chaque zone de fiche est affectée d'un code adresse (Documentaliste, 1971)

- la zone est l'emplacement de longueur variable qui suit un code et qui est destiné à contenir l'information (Bye, 1973) ;

- les indicateurs de zone définissent les zones sur lesquelles on veut faire porter la recherche (Chaumier, 1971).

\section{FORMATTING}

- structuration (des données) Unisist) ;

— mise en forme, mise en page, « édition $»$ (Ginguay, 1972).

\section{INDEXING AND ABSTRACTING SERVICES}

- services d'analyse et d'indexation (BIU, $\mathrm{n}^{\circ} 3,1973$ );

- services de résumés analytiques et d'indexation (BIU, $\left.\mathrm{n}^{\circ} 2,1974\right)$;

- d'autres revues bibliographiques: Chemical Abstracts, Biological Abstracts (Centre de documentation, CNRS (Paris), 1974).

\section{INDEXING JOURNAL}

— journal d'index (Unisist) ;

- bulletin bibliographique (Chaumier, 1971);

- les index imprimés (KWIC par exemple) (BS 101, 1971).

\section{INFORMATION RETRIEVAL}

- recherche documentaire, recherche d'information(s) (Ginguay, 1972) ;

- recherche de l'information, repérage de l'information, localisation de l'information (Pollet, 1974) ;

- la récupération de l'information (BIU, vol. $2, \mathrm{n}^{\circ} 3,1974$ );

- les méthodes de mise en mémoire et de restitution (BIU, vol. $2, \mathrm{n}^{\circ} 2,1974$ ).

INTERACTIVE (voir CONSERVATIONAL)

INTEREST PROFILE (voir aussi PROFILE DESIGN)

— profil d'intérêt (d'Olier 1967) (Unisist);

- les profils d'intérêt (Chaumier, (1971);

- profil d'intérêt ou profil documentaire BS 101, Suppl. 1975). 


\section{LITERATURE}

- le volume de la littérature scientifique et technique (d'Olier, 1967);

- documentation (DI 1971);

- références extraites de la presse scientifique mondiale (BS 101, Suppl. 1975).

\section{LITERATURE SEARCH}

- recherche documentaire (DDP 1970);

- recherches bibliographiques (SODEXPORT, France, 1972).

\section{LOGICAL OPERATOR (aussi LOGICAL CONNECTIVE,} LOGICAL CONNECTOR)

- opérateur logique (Ginguay, 1972);

- les fonctions logiques ET, OU, NON (Meta, 1971);

- les relations ET, OU et NON (intersection, réunion et exclusion de classes) (Bonnelly, 1973) ;

- les opérateurs logiques ET, OU, SAUF (Chaumier, 1971) ;

- opérateurs booléens classiques (ET, OU, SAUF) (Dusoulier, 1971).

\section{LOG ON, LOG OFF (voir SIGN ON, SIGN OFF)}

\section{MACHINE-READABLE}

- exploitable, assimilable par une machine (Ginguay, 1972) ;

- lisible par machine (BIU 1974/2).

\section{MATCH (voir SEARCH AGAINST)}

\section{OFF-LINE}

- autonome, indépendant ; traitement en différé (Ginguay, 1972) ;

- autonome, non connecté : se dit d'une unité ou d'un calculateur périphérique non connecté à une unité centrale ou à un calculateur central; se dit aussi de l'opération d'entrée de données ou de sortie de résultats, exécutée sur une unité périphérique non connectée (BT 155).

\section{ON-LINE (voir aussi TIME-SHARING)}

- direct, en direct (Unisist) (Ginguay, 1972) ;

- en ligne (BS 101);

- la consultation en accès direct par terminal (Meta, 1973);

- en ligne, connecté : se dit d'une unité ou d'un calculateur périphérique connecté à une unité centrale ou à un calculateur central; se dit aussi de l'opération d'entrée ou de sortie exécutée sur une unité périphérique connectée à l'unité centrale ou au calculateur central (BT 155).

\section{PRINTOUT}

- un bulletin d'imprimante individuel par semaine pour chaque profil; listeréponse (ISTC II-5, 1970) ;

- sorties graphiques, sorties chiffrées (Mathelot, 1969); 
- vidage sur imprimante (Ginguay, 1970) ; sortie sur imprimante (Ginguay, 1972);

- documents de sortie imprimés à l'aide d'une IBM 1403/3 (BS 101, 1970) ;

- la sortie imprimante (Chaumier, 1971);

- le listing est composé de fiches cartonnées de $100 \times 150 \mathrm{~mm}$ à raison de deux dans la largeur de la page (Deroche, 1973) ;

- une copie du feuillet de références; dans le document-réponse (mémoire présenté par A.-M. Boilard à l'Institut national des techniques de la documentation, Paris, 1974);

- listages mécanographiques, listings mécanographiques (Pollet, 1974).

\section{PROFILE DESIGN}

- rédiger des profils documentaires (Deroche, 1973);

- construire [...] élaborer un profil d'intérêt (Bonnelly, 1973).

\section{RECALL}

- pouvoir, taux de rappel (Wolff-Terroine, 1969);

- exhaustivité (Van Dijk, 1969).

\section{REFERENCE (aussi : CITATION)}

— référence (Unisist) ;

- référence répondant au profil d'intérêt ; références rangées par ordre de numéros croissants; on transfère à l'imprimante les références bibliographiques (d'Olier, 1967) ;

- les signalements d'articles ou de livres (Documentaliste, 1971);

- les références des articles, thèses, [...] ; signalement (y compris le résumé) (BS 101, 1974);

- les signalements des documents retenus (Poincaré, 1973);

— «éléments de citation» (c'est-à-dire les descriptions bibliographiques) (Unisist).

\section{REFERENCE WORK}

- recherche bibliographique (VTBB, 1969).

\section{REFERENCE WORK(S) (aussi : REFERENCE AIDS, REFERENCE TOOLS)}

- ouvrages de référence (VTBB, 1969);

- usuels : l'ensemble des livres et autres documents d'une bibliothèque conservés en vue de la consultation immédiate et rapide, et disponibles en tout temps à la bibliothèque, en particulier dans la salle de lecture et de consultation; se dit aussi d'ouvrages de référence d'un usage fréquent, comme les dictionnaires, les bibliographies, les annuaires, etc. (VTBB, 1969);

- outils documentaires (bulletins et index) (Documentaliste, 1971).

\section{REFERRAL}

- services d'inventaire(s), services signalétiques; services d'information sur les organismes documentaires (Unisist); 
- centre de référence (OCDE, 1971) ;

- centre d'aiguillage : dirige les demandes vers le système correspondant (BCE, 1974/4).

\section{REFORMAT}

- restructurer (Unisist) ;

- reconditionnement de l'information pour un public déterminé (Michel, 1973);

- remettre en forme, recomposer (Ginguay, 1972) ;

- reformaté, reformatage (Bye, 1973).

\section{RETRIEVE}

- BOLT choisira BOLT, mais éliminera BOLTZMANN; $\mathrm{x}$ conserve $\mathrm{xyz} ; \mathrm{x}$ couvrira xyz (Documentaliste, 1971) ;

- PHOTOSY récupère PHOTOSYNTHESIS ; les termes qui ont retrouvé la référence (Deroche, 1973) ;

- il est possible d'exiger la seule présence d'un mot dans un résumé pour assurer sa sélection (Documentaliste, 1971) ;

- les informations recueillies (Chaumier, 1971).

\section{SEARCH AGAINST (aussi MATCH)}

- le rôle du calculateur consiste à rapprocher les mots clés figurant dans les équations de recherche des mots clés caractérisant le contenu des documents (annonce du C.N.R.S. (Paris) dans Recherche, janvier 1972, p. 46) ;

- le profil sera confronté à la bande de données désirée ; comparer le profil aux bandes de données (mémoire présenté par A.-M. Boilard à l'Institut national des techniques de la documentation, Paris, 1974);

- le système est interrogé systématiquement sur ces questions (Chaumier, 1971).

\section{SEARCH EDITOR}

- rédacteur de recherches (Science Dimension 1972/4).

\section{SEARCH EXPRESSION}

- phrase logique ou équation de recherche (BS 101, Suppl. 1975).

\section{SEARCH FORMULATION}

- la mise en équation de la question (Van Dijk, 1969).

\section{SEARCH LOGIC}

- la logique d'interrogation choisie (Documentaliste, 1971).

\section{SEARCH STRATEGY}

— stratégie de recherche (Unisist).

\section{SELECTIVE DISSEMINATION OF INFORMATION}

- diffusion sélective de l'information (DSI (BIU 1973/2);

- la Diffusion sélective de l'information sur «profils» d'intérêt (D.S.I.) (BS) 1974). 


\section{SIGN ON}

- entrée en communication (Ginguay, 1972).

\section{SIGN OFF}

— fin de traitement (Ginguay, 1972);

- fin de message (DI 1971).

\section{STORAGE}

- stockage, rangement, mémorisation (Ginguay, 1972);

- enregistrement, emmagasinage (Unisist) ;

- les méthodes de mise en mémoire et de restitution (BIU 1974/2).

\section{TAPE SERVICES}

- le fonds documentaire INSPEC (Michel, 1973);

- fichiers documentaires sur bandes magnétiques (Van Dijk, 1969);

- fichiers (Compendex, Chemical Abstracts Condensates, etc.) (Poincaré, 1973).

\section{TERMINAL}

- poste terminal, terminal (Ginguay, 1972);

- plusieurs terminaux sont branchés sur un tronçon de ligne commun (Poincaré, 1973) ;

- unité terminale d'affichage; terminal d'interrogation; la console du terminal (Documentaliste, 1971) ;

- terminal : organe d'entrée ou de sortie relié à l'ordinateur par une transmission de données quelconque (BT 155).

\section{TIME-SHARING (voir aussi BATCH PROCESSING)}

- travail en partage de temps, exploitation en temps partagé (par utilisation collective d'un ordinateur) (Ginguay, 1972);

- partage de temps, (travail en) temps partagé : mode de traitement de l'information dans lequel plusieurs utilisateurs exécutent sur le même ordinateur des travaux indépendants, des tranches de temps étant affectées à chaque utilisateur, qui néanmoins peut suivre son propre rythme de travail (BT 155).

\section{TRUNCATION}

- par troncation de radicaux, « herbesan » pour herbe de santé (la Banque des mots, 6, 1973) ;

- utiliser des troncatures pour les radicaux communs à plusieurs mots (Deroche, 1973) ;

- tronquer (Ginguay, 1972) ;

- troncation, coupure (Ginguay, 1973) ;

- masque : recherche sur racines ; ex. : TRANSISTOR (Documentaliste, 1971).

USER

- utilisateur, usager, abonné, client (Ginguay, 1972). 


\section{WEIGHTING}

- en pondérant les descripteurs, on attribue une cote de classement à la réponse ; indice de pondération logique (ISTC II-5, 1970).

\section{WORD AUTHORITY LIST (aussi : CONTROLLED VOCABULARY)}

- listes de mots clés généralement classés dans l'ordre alphabétique et qui représentent le vocabulaire défini et autorisé pouvant être employé pour l'indexation des documents (Chaumier, 1971)

- termes autorisés, vocabulaire déterminé (Documentaliste, 1971)

- vocabulaire contrôlé (Dusoulier, 1971) (Marlot, 1973)

- un vocabulaire contrôlé ou thésaurus (B S 101, Suppl. 1975)

RoBert SERRÉ

\section{SOURCES}

Unisist

Documentaliste, 1971

Bonnelly, 1973

Bye, 1973

Chaumier, 1971

Deroche; $₫ 973$

Dusoulier, 1971

Ginguay, 1972

Marlot, 1973

Mathelot, 1969

Michel, 1973

d'Olier, 1967

Poincaré, 1973
Unisist, «Etude sur la réalisation d'un système mondial d'information scientifique », Paris, Unesco, 1971.

Unisist, «Conférence intergouvernementale pour l'établissement a'un système mondial d'information scientifique », Rapport final, Paris, Unesco, 1971.

Documentaliste, numéro spécial, 1971 : «L'automatisation documentaire en France et dans les pays d'expression française $»$.

Claude Bonnelly, «La référence automatisée dans les bibliothèques d'université et de recherche $»$, Documentation et Bibliotheques, vol. 19, 1973, p. 26-33.

Béatrice Bye, «Le système d'automatique documentaire du Centre de documentation des sciences humaines SPLEEN », Documentaliste, vol. $10, \mathrm{n}^{\circ} 2,1973$, p. 66-76.

Jacques Chaumier, «Les techniques documentaires 》, Que saisje? n० 1419 , Paris, PUF, 1971, $128 \mathrm{p}$.

Marie-Esther Deroche, «Une expérience d'interrogation des bandes magnétiques de Chemical Abstracts Service pour un groupe de chercheurs travaillant sur la photosynthèse », Documentaliste, vol. 10, $\mathrm{n}^{\circ} 3,1973$, p. 119-125.

N. Dusoulier et al., \& PASCAL, un nouveau service de gestion de la documentation $»$, Automatisme, vol. $16, \mathrm{n}^{\circ} 10,1971$, p. 537-542.

Michel Ginguay, Dictionnaire de l'informatique anglais-fransais, Paris, Masson, $3^{\circ}$ éd., 1972.

L. Marlot, «Un procédé moderne d'information : le système PASCAL, la Revue polytechnique (Genève), ${ }^{\circ} 1319$, novembre 1973, p. 1177-1181.

Pierre Mathelot, «L'informatique », Que sais-je? $\mathrm{n}^{\circ} 1371$, Paris, PUF, 1969, 128 p.

J. Michel, «Vers un réseau national de documentation », Automatisme, vol. 18,1973 , p. $180-184$.

J. d'Olier, «Aperçu sur les problèmes actuels de la documentation scientifique et technique $»$, Progrès scientifique, 114, 1967, p. 23-67.

F. Poincaré, «La recherche documentaire par ordinateur; le réseau de téléinformatique spatiale $»$, l'Onde électrique, mai 1973, p. $159-162$. 
Pollet, 1974

Van Dijk, 1969

Wolff-Terroine, 1969

AT

BCE

BIU

BS 101

BS 101, Suppl. 1975

BT 155

CETTF 1972

DDP

DI

ISTC II-3, 1970

ISTC II-5, 1970

OCDE, 1971

VTBB, 1969
Ray J. Pollet, « Lexique anglais-français du microfilm », Meta, vol. $19, \mathrm{n}^{\circ} 3,1974, \mathrm{p} .158-164$.

Marcel van Dijk et $\mathrm{G}$. Van Slype, le Service de documentation face à l'explosion de l'information, Paris, les Editions d'Organisation, $1969,265 \mathrm{p}$.

M. Wolff-Terroine et al., «Evaluation d'un système documentaire $»$, Information et documentation, juin 1969, p. 21-28.

l'Actualité terminologique (Ottawa).

Bulletrn des Communautés européennes (Bruxelles).

Bulletin d'information de l'UNISIST (Paris).

Bulletin signalétique, Section 101, Science de l'information, documentation.

Bulletin signalétique, Section 101, Supplément de janvier 1975.

Bulletin de terminologie, $\mathrm{n}^{\circ} 155$, «Arrêtés de terminologie de la République française », Ottawa, Information Canada, juillet 1974.

Comité d'étude des termes techniques français, Termes techniques français. Essai d'orientation de la terminologie, Paris, Hermann, 1972.

Claude Cani:lle et Michel Dehaine, Dictionary of Data Processing. Dictionnai"e de l'informatique, Toronto, Harrap, 2 vol. 1970.

Robert Dubuc et al., Dictionnaire anglais-français françaisanglais ae l'informatique, Québec, Dunod, 1971, 214 p.

Conseil des sciences du Canada, l'Information scientifique et technique au Canada, Etude spéciale $\mathrm{n}^{\circ} 8$, partie II, chap. 3, les Universités, Ottawa, 1970.

Conseil des sciences du Canada, l'Information scientifique et technique au Canada, Etude spéciale $\mathrm{n}^{\circ} 8$, partie II, chap. 5, les Techniques et les sources, 1970.

Examens des politiques nationales de l'information scientifique et technique, Canada, Paris, OCDE, 1971, $177 \mathrm{p}$.

Paule Rolland-Thomas et al., Vocabulaire technique de la bibliothéconomie et de la bibliographie, Montréal, ACBLF, 1969, $187 \mathrm{p}$. 